\title{
REVISTA PERUANA DE MEDICINA EXPERIMENTAL Y SALUD PÚBLICA: SU IMPORTANCIA EN LA DIFUSIÓN DE LA CIENCIA DESDE EL PERÚ
}

\author{
REVISTA PERUANA DE MEDICINA EXPERIMENTAL Y SALUD PÚBLICA: HIS IMPORTANCE IN THE \\ DISSEMINATION OF SCIENCE FROM PERU
}

César Cabezas ${ }^{1, a}$, Hugo Arroyo-Hernández ${ }^{1, b}$, Lely Solari ${ }^{1, c}$

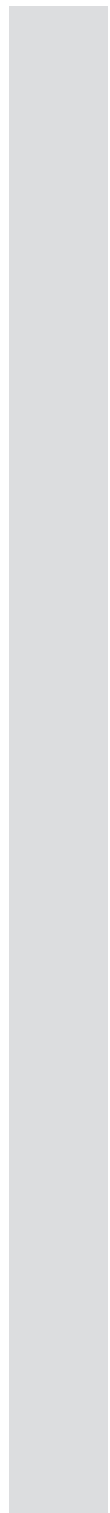

El esfuerzo por plasmar de manera escrita las evidencias científicas generadas en salud pública y medicina, y su difusión, se inició en octubre de 1942 mediante la Revista de Medicina Experimental, editada, en ese entonces, por los doctores Telémaco Batistini y Carlos Gutierrez-Noriega; fue una revista semestral que estuvo vigente hasta 1960. Desde sus albores, esta revista incluyó artículos de sumo interés para la salud pública nacional, como la peste, la leishmaniosis, la leptospirosis, la enfermedad de Carrión, la viruela, la enfermedad de Chagas, la malaria, la susceptibilidad de vectores al DDT, estudios sobre la cocaína y el cocainismo, el cáncer, la silicosis, y el yodo como micronutriente, en lo que denominaríamos primera época, estudios que incluían a la observación en campo y la experimentación como métodos.

En 1997 se marca el reinicio de la edición, como segunda época, con la misma línea editorial de la primera; en el editorial del primer número de ese año se menciona que: La Revista de Medicina Experimental (segunda época) aparece en momentos en que nuestro país, como muchos países latinoamericanos, sufre la presencia de enfermedades emergentes y reemergentes...II desde entonces, y hasta la actualidad, los problemas de salud siguen siendo vigentes y aun más complejos debido a factores tan variados como las inequidades, la globalización y el cambio climático, temas incluidos - al igual que antes- como parte del contenido de las publicaciones en las versiones actuales de la Revista ${ }^{(1,2)}$

Para reflejar el contenido y la orientación que siempre tuvo la Revista, el año 2002 pasó a denominarse Revista Peruana de Medicina Experimental y Salud Pública, de pronto, una denominación de amplio espectro, sin embargo, necesaria y pertinente en su momento. El año 2010 es indizada a Scopus y Medline, constituyendo un hito histórico, dado que es una de las dos únicas revistas científicas en salud indizadas en el país ${ }^{(3,4)}$. Desde entonces, el número de artículos recibidos para ser publicados se ha incrementado pasando de 798 artículos recibidos durante los años 2010 al 2012 a 1074 artículos recibidos del 2013 hasta agosto del 2015 con un promedio de artículos recibidos por año superior a 350, durante el 2013 y 2014.

El incremento en el número de artículos recibidos también nos ha llevado a adoptar nuevas plataformas de gestión editorial como el Open Journal System (OJS) un software de código abierto, con la finalidad de reducir el tiempo y el esfuerzo dedicado al manejo exhaustivo de las tareas que involucran la recepción, la revisión por pares y la edición de una publicación seriada; ello permitirá simplificar los procesos de edición y garantizará la publicación en línea y anticipada. Este sistema ha estado sometido a pruebas para lograr tener una plataforma amigable para nuestros lectores e investigadores que decidan enviar sus artículos a la Revista y que estará disponible para fines del 2015. Asimismo, junto con las actuales exigencias de publicación científica se ha gestionado el uso del DOI (Digital Object Identifier) para lograr identificar digitalmente los artículos publicados en nuestra revista sin importar

\footnotetext{
1 Revista Peruana de Medicina Experimental y Salud Pública. Instituto Nacional de Salud. Lima, Perú.

Editor general; ${ }^{\mathrm{b}}$ editor científico; ${ }^{\mathrm{c}}$ Comité Editor

Recibido: : 16-09-15 Aprobado: 16-09-15
}

Citar como: Cabezas C, Arroyo-Hernández H, Solari L. Revista Peruana de Medicina Experimental y Salud Pública: su importancia en la difusión de la ciencia desde el Perú [editorial]. Rev Peru Med Exp Salud Publica. 2015;32(3):421-2. 
su URL. Otra gestión importante ha sido la adquisición y uso de un software para comprobar la originalidad de los textos recibidos, lo que permite al equipo editor la detección de plagio.

Sibien nos debemos a nuestro público fundamentalmente de habla hispana, es también un objetivo a corto plazo el aceptar y publicar contenidos en idioma inglés, pues la publicación de artículos en este idioma tienen mayor visibilidad en la comunidad científica internacional lo cual podría mejorar los indicadores de citación de la Revista. Actualmente se están evaluando propuestas que permitan cumplir con el propósito de contar con una publicación científica peruana que dé visibilidad internacional a las investigaciones realizadas por peruanos y extranjeros, este es un hito importante, sin dejar de expresar que tan importante como ello es contar con un medio para llevar información con evidencia científica actualizada a quienes lidian por la salud de la población en los lugares más remotos de nuestro país y de la Región.

Debemos reconocer que la Revista todavía está en una etapa de consolidación y hay aún mucho camino por recorrer, siendo una de las metas su indización en ISI web of Science, una base de datos privada de la editora Thompson y Reuters, ello permitirá a la Revista obtener un indicador denominado factor de impacto. La comunidad científica internacional determina sus preferencias de publicaciones de acuerdo con este factor, por ello, es un reto para los próximos años que la revista logre esta indización y tenga sostenibilidad en el tiempo. La única revista peruana que figura en ISI, la Revista Latinoamericana de Crítica Literaria, es editada en la Universidad de Tufts, en Estados Unidos de Norteamérica y su último número corresponde al 2014, ante esta situación, es un reto que nuestra Revista sea parte de ISI en el campo científico de la salud.

La experiencia obtenida en la gestión de una revista científica peruana, financiada por una institución pública, como es el Instituto Nacional de Salud del Ministerio de Salud, muestra que la trascendencia de esta publicación se debe, en gran parte, a la continuidad editorial, que junto con otros indicadores, contribuyen al fortalecimiento de la institucionalidad, tan necesaria en el Perú. Un país con instituciones sólidas garantiza también la democracia. Sin embargo, debemos mencionar también, que el ser una de las pocas revistas indizadas, no constituye una completa satisfacción si se da una mirada más integral como país. Requerimos de más revistas científicas especializadas e indizadas que respondan a la necesidad actual de difundir una mayor producción científica y es, a su vez, un reto comprometernos a ese propósito de manera conjunta con todas las instituciones, tanto públicas como privadas, que desarrollan investigación y tienen la necesidad de difundir los conocimientos generados, para que puedan tener una incidencia en políticas públicas y acciones que ayuden a mejorar la salud en nuestras poblaciones a las que nos debemos como entidad pública.

\section{REFERENCIAS BIBLIOGRÁFICAS}

1. Cabezas C, Mayta-Tristán P. Evolución y perspectivas de la Revista Peruana de Medicina Experimental y Salud Pública, 1945-2008. Rev Peru Med Exp Salud Publica. 2008;25(2):167-68.

2. Burstein Z. La Revista Peruana de Medicina Experimental y Salud Publica en el año 2009. Rev Peru Med Exp Salud Publica. 2009;26(1):3-4

3. Burstein Z. Revista Peruana de Medicina Experimental y Salud Publica del Instituto Nacional de Salud. En Historia de la Salud Publica en el Perú. Academia Peruana de Salud Vol. 25 Sección II: Otros avances en la investigacion historica. Pag. 8983-96 JC Treiding Peru. SA 2013.

4. Burstein Z. Cabezas C. Avances y retos de la Revista Peruana de Medicina Experimental y Salud Publica al 2010. Rev Peru Med Exp Salud Publica. 2010;27(1):3-5 